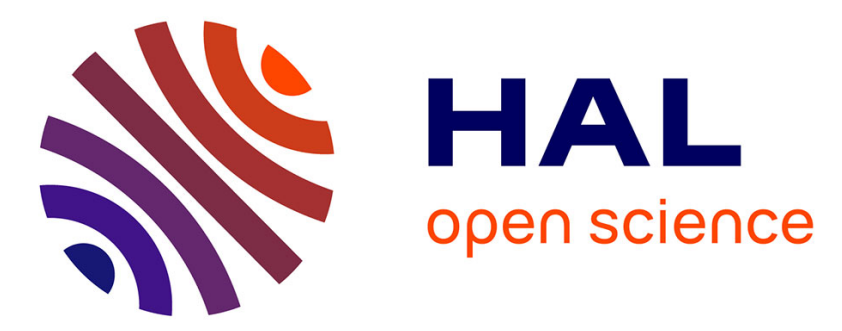

\title{
Mesoscopic Strain Fields Measurement During the Allotropic $\alpha-\gamma$ Transformation in High Purity Iron
}

\author{
Nicolas Bruzy, M. Coret, B. Huneau, G. Kermouche, M. Mondon, E.
} Bertrand, L. Stainier

\section{- To cite this version:}

Nicolas Bruzy, M. Coret, B. Huneau, G. Kermouche, M. Mondon, et al.. Mesoscopic Strain Fields Measurement During the Allotropic $\alpha-\gamma$ Transformation in High Purity Iron. Experimental Mechanics, 2019, 59 (8), pp.1145-1157. 10.1007/s11340-019-00525-z . hal-02317370v2

\section{HAL Id: hal-02317370 \\ https://hal.science/hal-02317370v2}

Submitted on 29 Oct 2019

HAL is a multi-disciplinary open access archive for the deposit and dissemination of scientific research documents, whether they are published or not. The documents may come from teaching and research institutions in France or abroad, or from public or private research centers.
L'archive ouverte pluridisciplinaire HAL, est destinée au dépôt et à la diffusion de documents scientifiques de niveau recherche, publiés ou non, émanant des établissements d'enseignement et de recherche français ou étrangers, des laboratoires publics ou privés. 


\title{
Mesoscopic strain fields measurement during the allotropic $\alpha-\gamma$ transformation in high purity iron.
}

\author{
N. Bruzy, M. Coret, B. Huneau ${ }^{1}$, G. Kermouche, M. Mondon², E. \\ Bertrand $^{3}$, and L. Stainier ${ }^{1}$ \\ ${ }^{1}$ Institut de Recherche en Génie Civil et Mécanique (GeM), UMR6183, \\ Ecole Centrale de Nantes, Université de Nantes, CNRS, Nantes, France \\ ${ }^{2}$ Laboratoire Georges Friedel (LGF), UMR5307, Mines de Saint-Etienne, \\ Université de Lyon, CNRS, Centre SMS, Saint-Etienne, France \\ ${ }^{3}$ Institut des Matériaux Jean Rouxel (IMN), UMR6502, Université de \\ Nantes, CNRS, Nantes, France
}

\begin{abstract}
The allotropic phase change from ferrite to austenite represents a moment of massive interplay between the microstructural and mechanical states of iron. The difference of compacity between the two phases induces a microplastic accommodation in the material at grain scale. However, mechanical heterogeneities resulting from the transformation process remain challenging to characterise due to the high temperature conditions it is associated with. We developed experimental equipment for in situ observation of $\alpha-\gamma$ and $\gamma-\alpha$ transformations. Images of the surface of an iron sample taken by an optical camera were used as input for a Digital Image Correlation (DIC) routine. Special care was taken to maximize image resolution to capture subgrain phenomena. Observations show that, at the mesoscopic scale, shear strain fields exhibit strong localisations that are evidence of transformations that are occurring. Keywords: Digital Image Correlation, High temperature, Allotropic transformation, Pure iron
\end{abstract}

\section{Introduction}

A wide field of study has been devoted to allotropic transformations of pure iron due to their key role in shaping the material properties of iron alloys. The direct $\alpha-\gamma$ and inverse $\gamma-\alpha$ transformations, which are the most frequently encountered during thermo-mechanical treatments of these alloys, have been given utmost consideration. It is well-known that under moderate temperature loading conditions both transformations are dominated by short-range 
diffusion. No chemical partitioning takes place in pure iron. Therefore atoms locally reorganise, which leads to the constitution of a rapidly moving interface between the parent and product phases.

The occurrence of allotropic transformations at the macroscale can be detected by performing dilatation tests on pure iron or ultralow carbon samples Liu et al. [2004]. The temperaturelongitudinal strain response of the material is quite linear everywhere except around $910^{\circ} \mathrm{C}$ where longitudinal strain tends to drop in the case of the $\alpha-\gamma$ transformation or to rise in the case of the $\gamma-\alpha$ transformation. This trend highlights the role of allotropic transformations on the mechanical state of the material. An explanation of this phenomenon lies in the respective crystal structure of each phase. Lattice parameters have been measured to be $0.365 \mathrm{~nm}$ for austenite and $0.291 \mathrm{~nm}$ for ferrite at $910^{\circ} \mathrm{C}$ Basinski et al. [1955. Elastoplastic strain fields develop around growing grains of the product phase Song et al. [2014] as a consequence of the combined changes in volume and compacity between the two lattices.

It seems beyond the realm of what is feasible to study the local reorganisation of atoms at the mesoscale. Instead, the observation of allotropic phase changes has to rely on indirect markers of their occurrence. Two kinds of markers can be imagined from the considerations of the previous paragraph: either the interface between the phases or the strain fields resulting from the transformation. The most straightforward approach is certainly to track the interface that is formed between parent and product phases. Zhang and Komizo Zhang and Komizo 2013 use a Laser Scanning Confocal Microscope (LSCM) apparatus to observe the appearance of new grain boundaries between ferrite and austenite through modifications on the surface morphology of the samples. This kind of interface identification can be performed at even finer scales. Zijlstra et al. Zijlstra et al. [2017] resort to a heating system inside a Scanning Electron Microscope (SEM), taking special care not to damage detectors. Electron Back Scattered Diffraction (EBSD) maps allow austenite to be distinguished from ferrite, thus leading to an estimation of the boundary motion velocity.

As pointed out above, another class of methods would hinge on the computation of local mechanical fields and their use as a tool to track transformation occurrence. This first requires a surface strain measurement method. Digital Image Correlation (DIC) is rather easy to implement in contrast to other available methods such as laser speckle interferometry. It has been proven reliable at very high temperatures Leplay et al. [2012], Mao et al. [2016]. In addition, it has been successfully applied to the characterisation of plastic activity inside grains of polycrystalline materials Gioacchino and da Fonseca [2015], Guery et al. [2016]. Nonetheless, to the knowledge of the authors, it has not been applied to the study of allotropic phase changes owing to the difficulty of acquiring accurate images of the surface of a sample at high temperatures (above $800^{\circ} \mathrm{C}$ ).

The methodology which is developed below aims at testing in conditions as close as possible to industrial processes. For instance, optical cameras will be used to circumvent the delay involved by scanning the surface with a SEM. The precision of the results is expected to improve continuously given the progression of optical systems Bosiers et al. [2006]. Finally, tests are performed on polycrystalline materials. Grain boundaries and triple points constitute favourable sites for nucleation of the product phase. Besides this, newly formed 
grains tend to respect an orientation relationship with one of the two parent grains along the boundary to minimise interfacial energy Strangwood 2012], which constrains the orientation of the product phase. Moreover, the variety of orientations in a polycrystal encourages strain heterogeneities. These are likely to increase the complexity of elasto plastic strain fields at the $\alpha-\gamma$ transition.

The two approaches presented previously are distinct and rather irreconcilable in the sense that although very useful, the information obtained by interface-based methods does not give strain distribution inside the material. Depositing a speckle pattern for DIC computations would alter the quality of LSCM photos or EBSD maps. Conversely, this patterning process hinders direct observations of the surface of a sample. The direction chosen in this work is the mechanics-oriented characterisation of allotropic transformation. Its objective is to come up with a new experimental set-up allowing in situ observation of this phenomenon, with a view to understanding how it interacts with the population of local defects. In section 2 , this set-up will be described. It will then be applied to the characterisation of the $\alpha-\gamma$ and $\gamma-\alpha$ transformations in a high purity iron sample in section 3 .

\section{Material and facilities}

\subsection{Material}

A high purity $\alpha$-iron sample was prepared by the cold crucible melting method Tumbajoy et al. 2018. The resulting metallic bar was forged and annealed to obtain an homogeneous microstructure with equiaxed grains. Annealing consisted in sealing the sample in a silica ampoule under a 200 mbar partial pressure of argon and heating it up to $800^{\circ} \mathrm{C}$ for 2 hours. The sample was then cooled in a furnace.

EBSD mapping was obtained using a JEOL JSM-6500F at $20 k V$ and an indexation step size of $5 \mu \mathrm{m}$. The objective was to obtain information on initial grain sizes and texture. To map the largest region possible, 176 acquisitions were performed and the corresponding "sub-maps" were stitched together. EBSD raw data were then processed with the MTEX toolbox Bachmann et al. 2011. Obtained orientations are displayed in Figure 1. They are colour-coded according to the given Inverse Pole Figure. Grain boundaries are computed with a tolerance of 5 degrees. Non indexed regions are left blank.
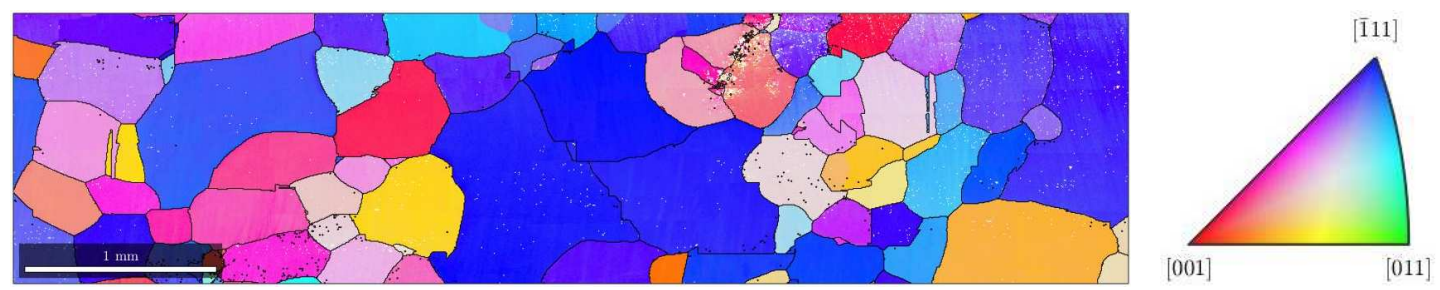

Figure 1: Grains orientations as obtained from EBSD measurements. Grains are colourcoded according to the adjacent key Inverse Pole Figure.

The microstructure is equiaxed and the mean initial grain size is around $250 \mu \mathrm{m}$, as 
confirmed by the grain sizes histogram shown in Figure 2. This microstructure is adequate for this work since it has to be much coarser than the image resolution of optical cameras to capture sub-grain phenomena. A typical recrystallisation $\gamma$-fiber texture Hutchinson 1989 can be expected given that the samples are annealed. Indeed, $48.1 \%$ of the measured orientations in Figure 1 are reported to be along that fiber with a tolerance of 10 degrees.

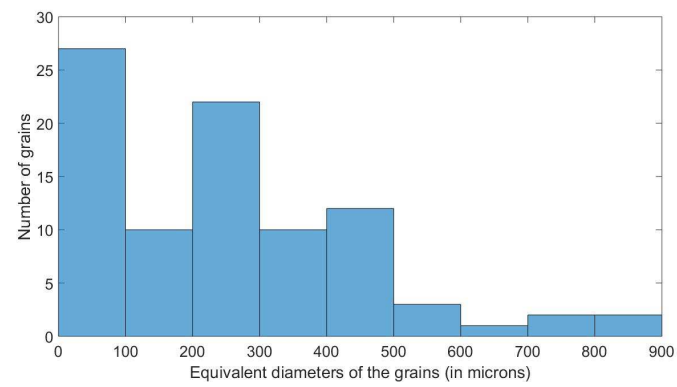

Figure 2: Histogram of equivalent grains diameters in the studied area.

\subsection{Description of the experimental device}

Figure 3 shows a photo of the experimental set-up with a side-view on the interior of the testing box. Samples are maintained in this box by two copper clamps, that are visible in Figure 3b. An operative clearance is preserved in such a way that one of the clamps can move freely and follow the sample thermal expansion movement. This prevents buckling of the sample during heating.

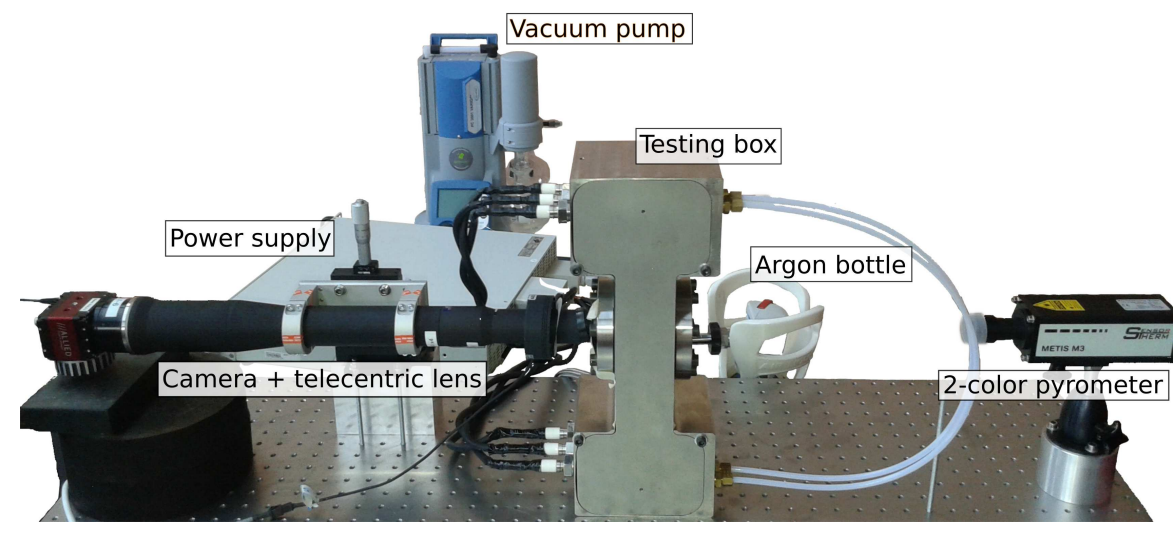

(a)

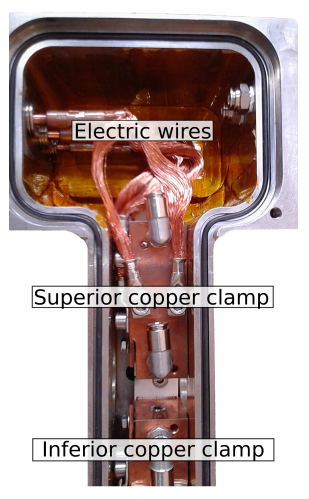

(b)

Figure 3: Photo of the experimental set-up and detailed view of the interior of the box.

Joule heating is preferred to other heating methods owing to its volumetric character and the easy access to the sample it provides. A TDK-Lambda Genesys 5kW power supply with 500A maximum current provides electrical current. The samples gripping area is inserted 
into a notch in the clamps. A screw is tightened in the clamps to maintain good electrical contact during the whole experiment. The copper clamps are massive enough to confine temperature variations to the sample, and thus prevent degradation of the equipment. In point of fact, the volume of one copper clamp is approximately $75000 \mathrm{~mm}^{3}$ while the volume of the sample is around $750 \mathrm{~mm}^{3}$.

Temperature is measured by means of a 2-colour pyrometer (SensorTherm METIS M322). Samples grain size are considered sufficient to justify not measuring temperature and kinematic fields on the same side of the sample. 2-colour measurements make the measured quantities independent from the intrinsic value of the emissivity of the sample Zhang and Machin 2009.

The calibration of the emissivity slope was performed using a batch of three iron samples. They were heated by the experimental equipment at a speed of approximately $1^{\circ} \mathrm{C} . \mathrm{s}^{-1}$. The occurrence of the ferrite-to-austenite transformation could be clearly detected from a thermal arrest in the temperature measured by the pyrometer. The difference between the measured temperature for the beginning of transformation and its reference value was used to fit the emissivity slope of the pyrometer.

High-quality sapphire windows (0-deg orientation, $0.2 \lambda$ flat) are used as observation windows in order not to alter pyrometer measurements and to reduce the effect of glass windows on the quality of the images Lyons et al. [1996].

\subsection{Optical system}

A high-resolution optical camera (Prosilica GT6600 from Allied Vision, 6576x4384 px resolution) records images. It is equipped with a $\mathrm{x} 4$ telecentric lens (Opto-Engineering TC $16 \mathrm{M}$ 009-F). Its magnification allows focusing on a region of interest of $9 \mathrm{~mm}$ by $6 \mathrm{~mm}$. A spatial resolution for imaging of $1.4 \mu \mathrm{m}$ is achieved with this optical system. The maximum framerate at full resolution is four images per second. Image acquisition is monitored by means of Vic-Snap software. It is synchronised with the temperature signal coming from the pyrometer so that the temperature value is recorded each time a photo is taken.

Care is taken to avoid chemical degradation of the samples. The box is cleaned of impurities before testing by pumping it down to a vacuum of 3 mbar using the rotary vane pump shown in Figure 3a. The samples are further immersed in an inert argon atmosphere to prevent oxidation. As pointed out in previous studies dealing with DIC at high temperatures Grant et al. 2009], a heat haze effect, i.e. a distortion of the images due to local variations of the refractive index of the surrounding gaseous atmosphere, may occur. Novak and Zok Novak and Zok 2011 use an air knife to reduce the negative effect of heat haze. In this study, a constant flow of argon was maintained during the tests so as to limit image distortions and thereby, no distortion is observed in the images as captured by the camera.

It is also necessary to avoid the influence of gray body radiation from the sample. As the sample is heated, it radiates a light from which energy increases with the wavelength. More 
precisely, the intensity of the radiation is governed by Planck's Law:

$$
I(\lambda, T)=\frac{2 h c^{2}}{\lambda^{5}} \frac{1}{e^{\frac{h c}{\lambda k T}}-1},
$$

where $\lambda$ is the wavelength, $T$ is the temperature, $c$ the speed of light, $h$ is Planck's constant and $k$ is Boltzmann's constant. On a practical level, the higher the wavelength, the more detrimental radiation is to image quality. It is possible to maintain good image contrast up to $1400^{\circ} \mathrm{C}$ with UV illumination Dong et al. [2014]. However, working in the blue wavelengths domain is sufficient for the range of temperatures involved in the study of the $\alpha-\gamma$ transformation Pan et al. 2011]. A blue LEDs ring provides light. A blue filter is put in front of the camera to cut-off higher wavelengths and restrict the gathering of information purely to the blue light reflected by the sample. One may consider the gray levels histograms in Figure 4 as proof of the viability of this lighting system. An acceptable contrast is preserved during testing, even at temperatures as high as $950^{\circ} \mathrm{C}$.

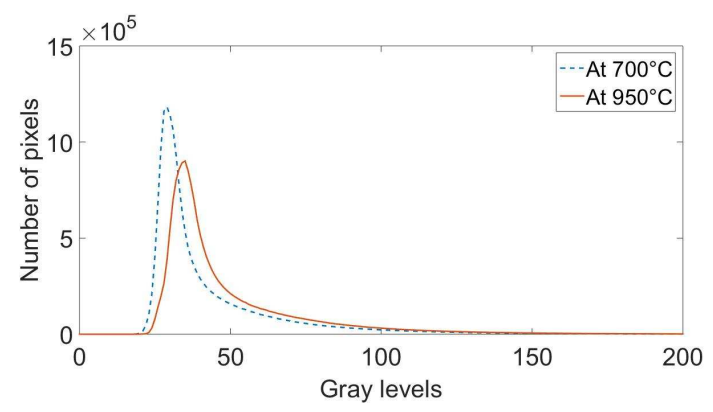

Figure 4: Gray levels histograms of two images taken at two different temperatures.

\subsection{DIC algorithm used}

FE-DIC computations are carried out using UFreckles software Réthoré [2018]. The following paragraph describes the main steps to obtain the displacement field in the region of interest. Let us consider two images, denoted by $f$ and $g, f$ being the reference image. Both are described by their gray values at each pixel. It is supposed that $g$ is the consequence of transforming points in $f$ according to a displacement field $u$. Conservation of the optical flow is assumed:

$$
f(x)=g(x+u(x)) .
$$

Two main difficulties prevent the determination of $u$ at this point: this scalar equation is not enough to compute the vector quantity $u$, and there may not be a unique correspondence between points in $f$ and $g$. The optical flow equation is reformulated in weak form to tackle these issues. Making additional use of a Taylor expansion, the correlation problem can be summed in the following minimisation problem:

$$
\min _{u} \iint_{\Omega}\|\delta u(x) \cdot \nabla g(x)+g(x+u(x))-f(x)\|^{2} d x .
$$


Non-pixel values are determined using a bi-cubic interpolation. Strain localisations are expected to appear in the experiments. Regularisation is performed to avoid the strain becoming excessively large for an element, thus impeding convergence of the DIC algorithm. As explained in Marty et al. [2015], an additional term is added to the functional to be minimised in the fashion of a Tikhonov regularisation:

$$
\min _{u} \iint_{\Omega}\|\delta u(x) \cdot \nabla g(x)+g(x+u(x))-f(x)\|^{2} d x+\omega\left(l_{c}\right) \iint_{\Omega}\|\nabla u(x)\|^{2} d x .
$$

This contribution is weighted by a parameter $\omega\left(l_{c}\right)$. The regularisation can be shown to act as a low-pass filter whose cut-off wavelength $l_{c}$ can be adjusted.

The displacement field is further discretised by constructing a mesh and using a finite element approximation of displacement. Q4 finite element shape functions are chosen in the same fashion as in Besnard et al. [2006]:

$$
u(x)=\sum_{\alpha, n} a_{\alpha n} \Psi_{n}(x) \mathbf{e}_{\alpha},
$$

where $a_{i j}$ are the discretisation parameters, $\Psi_{i}$ the shape functions, and $\mathbf{e}_{\alpha}$ the considered direction. The minimisation problem (4) is non-linear. Therefore it is solved iteratively. It can be shown that after discretisation, it culminates in a series of linear problems with the displacement increment as an unknown: find the parameters $a_{\alpha n}$ such that

$$
\begin{array}{r}
\sum_{n} \sum_{\alpha} a_{\alpha n} \iint_{\text {elem }}\left(\Psi_{n}(x) \Psi_{m}(x) \partial_{\alpha} g(x) \partial_{\beta} g(x)+\omega \partial_{\beta} \Psi_{m}(x) \partial_{\alpha} \Psi_{n}(x)\right) d x= \\
\iint_{\text {elem }}(f(x)-g(x)) \Psi_{m}(x) \partial_{\beta} g(x) d x,
\end{array}
$$

where the notation $\nabla \mathbf{h}(\mathbf{x}) \cdot \mathbf{e}_{\alpha}=\partial_{\alpha} h(x)$ is adopted.

The DIC procedure then takes the following form:

- an initial displacement increment guess is made. However, a Taylor approximation is used. Hence, displacement has to remain small. A coarse graining strategy is adopted: calculations are performed on supergrids of $2 \times 2,4 \times 4,8 \times 8$ cells, etc, and the obtained displacements are used as starting points for the iterations on the finer grids.

- quantities of interest are computed for the current iteration. In particular, image gradients are obtained thanks to a finite difference scheme.

- if the increment of displacement is sufficiently small, computations are stopped. Otherwise, the process is repeated until convergence.

\subsection{Samples design}

The geometry of the tested sample is shown in Figure 5. Electrical resistance is inversely proportional to the section. The fillets thereupon aim at ensuring that temperature is maximal in the center of the sample. Besides, the central part is long enough for the temperature 
to establish a zone where it is homogeneous. The $\alpha-\gamma$ transformation should then initiate in this zone during heating. Heterogeneities in the mechanical state of the material drive the transformation process since the temperature is the same everywhere. This leads to what can be characterised as a "stationary" transformation behaviour.

A gradient of temperature is likely to develop in the rest of the sample. The transition temperature for transformation is crossed only in a narrow slice of the sample at any given time. It can be expected that a transformation front will propagate towards the extremities of the sample, thus leading to the characterisation of a "transient" transformation behaviour. It has to be noted that temperature measurements during testing are only performed at the center of the sample, where temperature is maximal. The diameter of the pyrometer spot is $0.9 \mathrm{~mm}$, which is much smaller than the sample width.

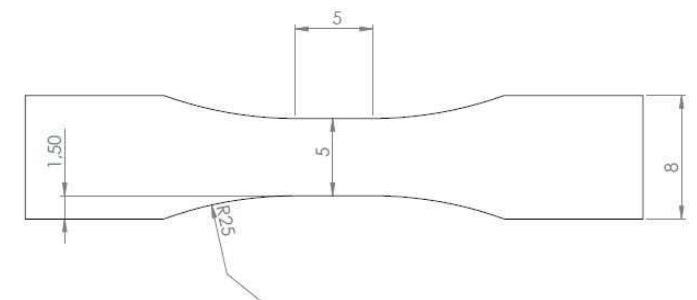

Figure 5: Drawing of the sample (dimensions in $\mathrm{mm}$ ). The sample thickness is $1 \mathrm{~mm}$.

\subsection{Samples preparation}

Performing image correlation requires the application of a speckle pattern. Following the recommendations of Dong et al. Dong et al. [2015], alumina-based painting was deposited on the surface of the samples with a fine-nozzle airbrush. A photo of the sample with the speckle pattern on it as captured by the optical acquisition system is displayed in Figure 6 . The minimal resolution for kinematic fields that can be attained from this pattern is around $50 \mu \mathrm{m}$.

The testing methodology is the following:

1. A first EBSD analysis is made;

2. The speckle pattern is deposited on the surface of the sample;

3. The sample is heated up to the transformation temperature then cooled to room temperature in the experimental equipment;

4. the sample is cleaned in an ultrasonic oscillated bath of ethanol after testing and another EBSD analysis is carried out. Grains morphologies and orientations in the final state are thus captured.

Three landmarks are made on the sample by micro-indentation to register experimental images and EBSD maps so that they match together. The width of these marks is around 
$5 \mu \mathrm{m}$. They are purposely placed far from the zone of interest to avoid introducing residual strains that may have an influence on the transformation onset. Their approximate positions are indicated by green squares in Figure 6. The blue rectangle corresponds to the area in which EBSD data and kinematic fields are represented. It covers a large portion of the sample's surface, as highlighted by the image.

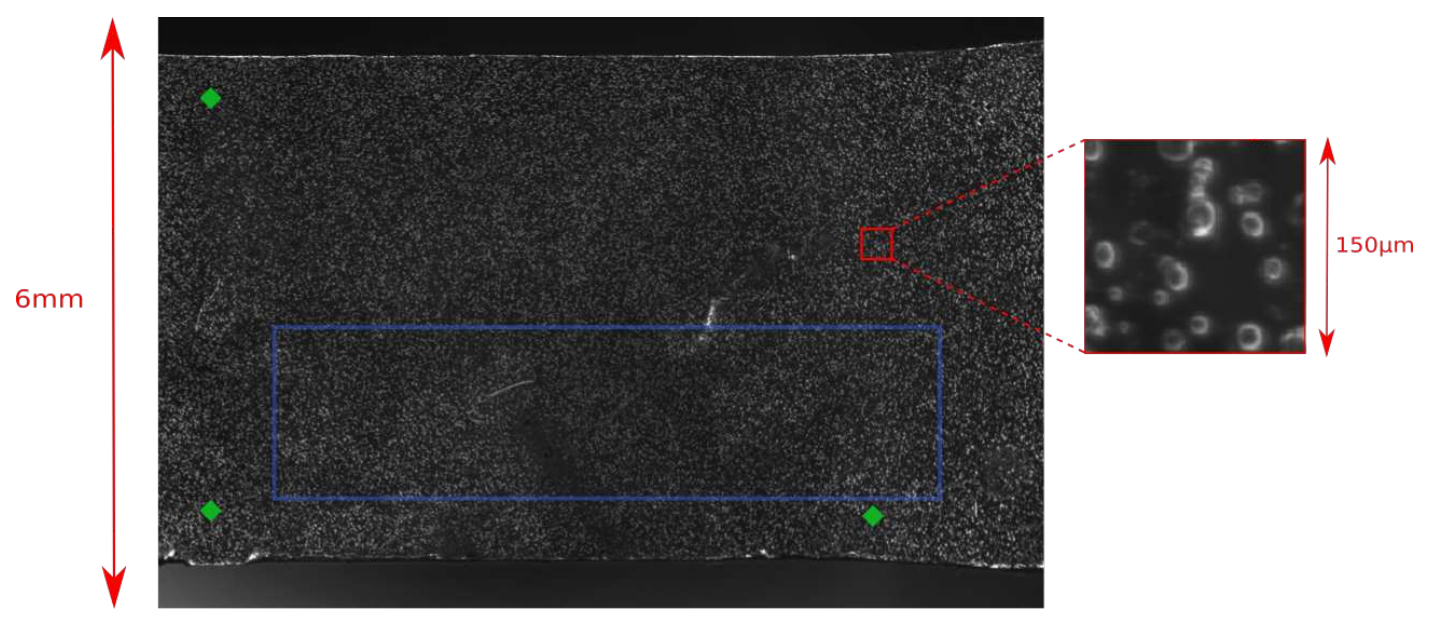

Figure 6: Image of the sample with the speckle pattern on it as taken by the camera. The blue rectangle indicates the zone where DIC results and EBSD maps are presented. The green squares indicate the approximate position of the landmarks used for registration.

\section{Application of the observation of ferrite-austenite transformation}

\subsection{Temperature time evolution}

The temperature follows the profile shown in Figure 7 during the test. Around the transformation region, the target loading consists of an increasing temperature ramp followed by a plateau and a decreasing temperature ramp. Another plateau is introduced before that at $650^{\circ} \mathrm{C}$ to check that parameters associated with image acquisition, namely exposition time and focusing, are adapted to the capturing of the image. Photos are taken between $t=50 \mathrm{~s}$ and $t=150 \mathrm{~s}$, which means that 400 images are available for post-processing at the end of the experiment.

Important temperature variations can be expected because the sample is thin. The maximum heating rate that can be attained with the set-up shown in Figure $3 \mathrm{a}$ and the sample geometry in Figure 5 is $500^{\circ} \mathrm{C} / \mathrm{s}$. However, heating rates are limited in practice by the framerate of the image acquisition system. 


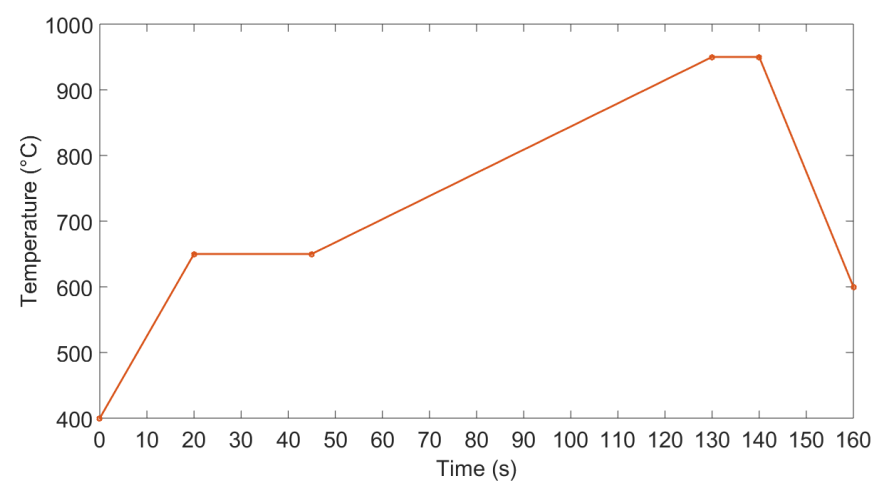

Figure 7: Temperature set point.

\subsection{Strain field evolution}

The finite element mesh used for DIC computations has a grid size of 25 pixels. The cutoff wavelength for regularisation is chosen to be the same as the grid size. Three levels of coarsening are considered, which means that a first calculation was performed with the surface of the cells increased by a factor of 16 , then by a factor of 4 . Convergence is attained once the norm of the increment of displacement is inferior to 0.01 pixel. Once displacement is known, the strain field is computed using the derivatives of the finite element shape functions. No smoothing takes place or intervenes in this process.

Elasto plastic strains accommodating the compacity difference between the phases have principal directions that are not necessarily aligned with the lab frame, depending on the orientation of the forming product grain and of the surrounding parent grains. Consequently, shearing can be expected when transformation occurs. We thus choose to compute the Maximum Shear Strain (MSS) quantity, defined as:

$$
s_{\text {max }}=\sqrt{\left(\frac{E_{x x}-E_{y y}}{2}\right)^{2}+E_{x y}^{2}},
$$

where $E$ is the 2D Green-Lagrange strain tensor. One advantage of this definition is that the $E_{x x}-E_{y y}$ term eliminates thermal expansion.

Figure 8 shows the evolution of this quantity during the test in the zone delimited in Figure 6. The initial microstructure extracted from EBSD measurements is superimposed on the correlation results. The temperature at which strain localisations first appear is called $T_{\alpha-\gamma}$ in the legend of the figure. This definition is of course only valid at the center of the sample and it ignores temperature gradients.

During heating and before transformation, the sample exhibits a thermal expansion deformation that is purely isotropic: MSS equals zero (Figure 8a). Strain localisations start appearing (Figure 8b) when the transformation begins. As expected, transformation first takes place in the central zone of the sample where temperature is maximal and homogeneous (Figure 8c to 8f). 
The fact that the transformation induces strain localisations of a significant magnitude is coherent with modelling studies dealing with diffusive transformations Barbe and Quey 2011. Moreover, the experimental data seems to verify that the product phase also undergoes deformation since MSS does not evolve much once the material is transformed.

Soon a transformation front develops and progresses in the opposite direction to the temperature gradient (Figures $8 \mathrm{~g}$ to 81). The temperature plateau is reached after some time. Figure $8 \mathrm{~m}$ depicts the maximum advancement of the transformation front.

During cooling, a front propagates from the extremities of the sample to its center (Figure $8 \mathrm{n}$ to 8r once the temperature allows the occurrence of $\gamma-\alpha$ transformation. As the field of study is not centered around and with respect to the sample, the front coming from the left of the sample is not visible before Figure $8 \mathrm{~s}$. The two fronts are highlighted using white arrows in Figure 8t. These two fronts coming from both extremities of the sample eventually join. High plastic strains are recorded at this moment (Figure $8 \mathrm{u}$ ) due to orientation incompatibilities.

\subsection{Nucleation around a triple point}

Figure 9 provides a zoom on a region of the sample located around a triple point. This zone is indicated with a green square in Figure 8u. Again, the initial microstructure is superimposed on the correlation results. MSS can be viewed as a norm that quantifies mechanical perturbations induced by the transformation. The shear strain $E_{x y}$ field is represented here in order to distinguish between shearing directions.

Two shear strain extrema, highlighted by the dotted circles, develop inside the material between the two snapshots. It can be surmised from this observation that an austenite grain has nucleated near the zone marked by the black arrow, that is to say close to the triple point area. The development of strong shear strain is coherent with the necessity to accommodate the volume misfit between the phases elasto plastically. Both the ferrite grains at the top and the bottom of the image had to accommodate this misfit. The opposite signs of elasto plastic shear strains are a consequence of different initial orientations of the ferrite grains. Figure 10 provides a zoom on the EBSD maps in the studied area. The proposed transformation scenario around the triple point could lead to such an evolution in the microstructure of the sample. However, it is hard to draw conclusions on its validity as the final microstructure results from both the direct $\alpha-\gamma$ and the inverse $\gamma-\alpha$ transformations.

\subsection{Error quantification}

Local error for DIC computations can be defined as the gap between the gray values of the reference image and those of the other image warped by the calculated displacement. Using notations of part 2.4, it reads:

$$
e r r=\|g(x+u(x))-f(x)\|
$$

Figure 11 displays the mapping of this local error at the beginning of the temperature plateau, i.e. at $t=130 \mathrm{~s}$. 
Figure 11 shows that the local error stays under $5 \%$ in a very large part of the sample, which underlines the reliability of the method when it comes to computing kinematic fields during $\alpha-\gamma$ transformation. Moreover, the zones in which the error exceeds this $5 \%$ value can be related to the grain boundaries of the newly formed austenite. This behaviour can be expected given that grain boundaries act as discontinuities that interfere with the correlation routine. It should not be detrimental to the characterisation of allotropic phase change since, as shown in the previous section, relevant evidence of the transformation process is to be found in the strain localisations around the newly formed grains.

\subsection{Macroscopic behaviour}

Longitudinal and shear strains averaged over the whole domain of study are displayed in Figure 12. The periods of time during which $\alpha-\gamma$ and $\gamma-\alpha$ transformations take place are highlighted by a green overlay. Dashed lines separate the heating phase, the temperature plateau and the cooling phase.

Note that the first image used for DIC computations is supposed to be the reference state, which accounts for the zero value of longitudinal strain at the beginning. During the heating phase, longitudinal strain exhibits a regular increase due to thermal expansion followed by a sudden drop caused by transformation. During cooling, the inverse trend is obtained. Longitudinal strain decreases linearly but for a jump towards positive values when the $\gamma-\alpha$ transformation occurs. The macroscopic longitudinal strain matches the classical dilatation behaviour described in the Introduction, which provides a validation for the experimental procedure.

Shear takes non-zero values only after the onset of the $\alpha-\gamma$ transformation in agreement with previous assumptions. It undergoes a second drop at the $\gamma-\alpha$ transformation, which may be a consequence of the strong plastic strains remaining in the material after Figure 8 i. On average, they dominate slightly over the relaxation of internal stresses highlighted in Figure 8g.

\subsection{Post mortem EBSD and orientations evolution}

Figure 13 shows a mapping of grain orientations after testing. Only $17.4 \%$ of the orientations are within the $\gamma$-fiber texture (considering the same tolerance of 10 degrees as above), which indicates an evolution in the texture of the material. The EBSD mapping in Figure 13 also exhibits more intra-granular misorientations compared to the initial analysis. This is a consequence of the accumulation of dislocations that cause local rotations Wilkinson [2000], which confirms that the allotropic transformation has been accommodated elasto-plastically. In addition, Figure 13 shows the formation of acicular grains reminiscent of Widmanstätten ferrite Lee et al. 2002]. This structure indicates that displacive mechanisms were in operation during the $\gamma-\alpha$ transformation.

Minimal rotation angles can be computed point by point between initial and final states to better highlight the differences between the two. They are defined as the angle associated 
with the rotation:

$$
\Delta O=O_{i} \times O_{f}^{-1}
$$

where $O_{i}$ and $O_{f}$ are the orientation tensors in the initial and final states respectively. These angles are represented in Figure 14a.

Three zones, delimited by red dotted lines, can be roughly identified in Figure 14a;

- the zone to the right (1) corresponds to grains that were not reached by the transformation front. Consequently, the minimal rotation angle between the two states equals zero;

- in the central part (2), that is the zone of propagation of the front, areas with similar angular differences are quite large. Growth phenomena seem to have been dominant over nucleation events during $\alpha-\gamma$ transformation. This results in a microstructure with elongated grains.

- in the zone to the left (3), where fronts join, the presence of small grains relates to the accommodation of orientation incompatibilities.

Figure 14b displays a histogram of angular differences. It can be seen in this Figure that angle differences in the central zone of the field of study are comprised within a discrete set of angles. This specific distribution might be a clue for the identification of the austenite orientations that are selected during the transformation process, which will be the topic of a future work.

\section{Conclusion}

An experimental device that allows for the capturing of images of a sample without loss in image quality at temperatures as high as $900^{\circ} \mathrm{C}$ is proposed. Such images are used to perform a DIC study and extract the strain field evolution during heating and cooling of an iron sample. The microstructure of the sample is coarsened up to a mean grain size of $250 \mu \mathrm{m}$ so that even with an optical camera equipped with a telecentric length, the resolution is sufficient to study the evolution of the strain field at a subgrain scale. The iron samples are submitted to temperature ramps crossing the equilibrium $\alpha-\gamma$ and $\gamma-\alpha$ transition temperatures, with the heating rate being adjusted to take enough photos.

Maximal shear strain, that removes isotropic thermal strain, is shown to be a good indicator of transformation occurrence. It takes zero value in the absence of transformation and it marks the occurrence of phase changes through the appearance of strain localisations. During heating, the conditions of the present study dissociate two behaviours depending on whether the temperature is homogeneous in the zone of the sample reaching the transformation temperature. The adjacency of shear strain peaks during the first phase is shown to be a marker of the nucleation of a new austenite grain. The second phase corresponds to the propagation and stabilisation of a transformation front. A thorough study of the kinetics of front propagation might provide a simple tool for the evaluation of the mobility of the 
ferrite-austenite interface.

Transformation fronts propagate backwards during cooling, erasing most of the shearing, except at the centre of the sample where residual plastic straining remains. Post mortem analysis of grain orientations reveal that front propagation gives priority to growth events compared to front junction, which induces a lot of nucleation phenomena in an attempt to accommodate orientations misfit. At the macroscopic scale, the averaged longitudinal strain over the surface of the sample permits the retrieval of classical thermal expansion data, which increases confidence in the relevance of the obtained mesoscale fields.

As emphasised previously, the limitations in terms of temperature loading rate and resolution depend only on the optical equipment used. Consequently, it is expected that this study will pave the way for fine-scale studies of iron alloys submitted to extreme temperature conditions, e.g. during welding.

\section{Acknowledgements}

This work is part of the SMICE project, which was funded by BPI France under grant P113013-2660682. The authors are extremely grateful to Julien Réthoré for providing access to the UFreckles software and for giving precious advice on how to use it.

\section{Bibliography}

F. Bachmann, R. Hielscher, and H. Schaeben. Grain detection from 2D and 3D EBSD data - specification of the MTex algorithm. Ultramicroscopy, 111:1720 - 1733, 2011.

F. Barbe and R. Quey. A numerical modelling of 3D polycrystal-to-polycrystal diffusive phase transformations involving crystal plasticity. International Journal of Plasticity, 27: 823-840, 2011.

Z.S. Basinski, W. Hume-Rothery, and A.L. Sutton. The lattice expansion of iron. Proceedings of the Royal Society A, 1955.

G. Besnard, F. Hild, and S. Roux. Finite-element displacement fields analysis from digital images: application to Portevin-Le Châtelier bands. Experimental mechanics, 46:789 803, 2006.

J.T. Bosiers, I.M. Peters, C. Draijer, and A. Theuwissen. Technical challenges and recent progress in CCD imagers. Nuclear instruments and Methods in Physics Research A, 565: $148-156,2006$.

Y. Dong, H. Kakisawa, and Y. Kagawa. Optical system for microscopic observation and strain measurement at high temperature. Measurement Science and Technology, 25, 2014.

Y. Dong, H. Kakisawa, and Y. Kagawa. Developement of microscale pattern for Digital Image Correlation up to $1400^{\circ} \mathrm{C}$. Optics and Lasers in Engineering, 68:7 - 15, 2015. 
F. Di Gioacchino and J. Quinta da Fonseca. An experimental study of the polycristalline plasticity of austenitic stainless steel. International Journal of Plasticity, 74:92 - 109, 2015.

B.M.B. Grant, H.J. Stone, and M. Preuss. High-temperature strain field measurement using Digital Image Correlation. Journal of Strain Analysis, 44:263 - 271, 2009.

A. Guery, F. Hild, F. Latourte, and S. Roux. Slip activities in polycrystals determined by coupling DIC measurements with crystal plasticity calculations. International Journal of Plasticity, 81:249 - 266, 2016.

W.B. Hutchinson. Recrystallisation textures in iron resulting from nucleation at grain boundaries. Acta metallurgica, 37:1047-1056, 1989.

J. Lee, K. Shibata, K. Asakura, and Y. Masumoto. Observation of the $\alpha-\gamma$ transformation in ultralow-carbon steel under a high temperature optical microscope. ISIJ International, 42:1135-1143, 2002.

P. Leplay, J. Réthoré, S. Meille, and M. Baietto. Identification of asymmetric consitutive laws at high temperature based on Digital Image Correlation. Journal of the European Ceramic Society, 32:3949 - 3958, 2012.

Y.C. Liu, F. Sommer, and E.J. Mittemeijer. Abnormal austenite-ferrite transformation behaviour of pure iron. Philosophical magazine, 84:18:1853 - 1876, 2004.

J.S. Lyons, J. Liu, and M.A. Sutton. High-temperature deformation measurements using digital image correlation. Measurement Science and Technology, 36:64 - 70, 1996.

W.G. Mao, J. Chen, M.S. Si, R.F. Zhang, Q.S. Ma, D.N. Fang, and X. Chen. High temperature digital image correlation evaluation of in-situ failure mechanism: an experimental framework with application to $\mathrm{C} / \mathrm{SiC}$ composites. Materials Science $\mathcal{E} 3$ Engineering A, 665:26-34, 2016.

J. Marty, J. Réthoré, A. Combescure, and P. Chaudet. Finite strain kinematics of multi-scale material by digital image correlation. Experimental mechanics, 55:1641-1656, 2015.

M.D. Novak and F.W. Zok. High-temperature materials testing with full-field strain measurement: experimental design and practice. Review of Scientific Instruments, 82, 2011.

B. Pan, D. Wu, Z. Wang, and Y. Xia. High-temperature Digital Image Correlation method for full-field deformation measurement at $1200^{\circ} \mathrm{C}$. Measurement Science and Technology, 22, 2011.

J. Réthoré. Ufreckles, October 2018. URL https://doi.org/10.5281/zenodo.1433776.

S.J. Song, F. Liu, and Z.H. Zhang. Analysis of elastic-plastic accomodation due to volume misfit upon solid-state phase transformation. Acta materialia, 64:266 - 281, 2014. 
M. Strangwood. Phase Transformations in Steels, chapter Fundamentals of ferrite formation in steels. Woodhead Publishing Limited, 2012.

D.Y. Tumbajoy, X. Maeder, G. Guillonneau, S. Sao-Joao, S. Descartes, J.M. Bergheau, C. Langlade, J. Michler, and G. Kermouche. Microstructural and micromechanical investigations of surface strengthening mechanisms induced by repeated impacts on pure iron. Materials \& Design, 147:56-64, 2018.

A.J. Wilkinson. Electron Backscatterd Diffraction in Materials Science, chapter Measuring strains using electron backscattered diffraction. Springer, 2000.

X.F. Zhang and Y. Komizo. In situ investigation of the allotropic transformation in iron. Steel Research International, 84:751 - 760, 2013.

Z. Zhang and G. Machin. Experimental Methods in the Physical Sciences, chapter Overview of Radiation thermometry. Elsevier, 2009.

G. Zijlstra, M.S.B. van Daalen, D.I. Vainchtein, V. Ocelik, and J.T.M. De Hosson. Interphase boundary motion elucidated through in-situ high temperature electron back-scatter diffraction. Materials and design, 132:138 - 147, 2017. 


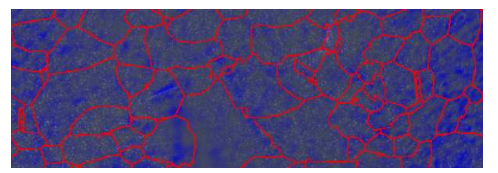

(a) $\mathrm{t}=99.75 \mathrm{~s}, \mathrm{~T}=T_{\alpha-\gamma}-1 K$

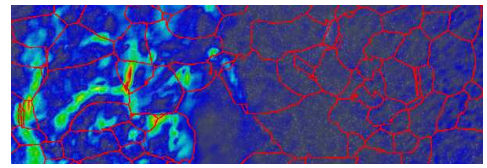

(d) $\mathrm{t}=101.25 \mathrm{~s}, \mathrm{~T}=T_{\alpha-\gamma}+6 K$

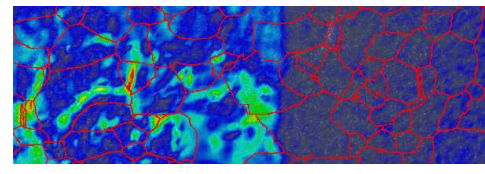

(g) $\mathrm{t}=105 \mathrm{~s}, \mathrm{~T}=T_{\alpha-\gamma}+18 K$

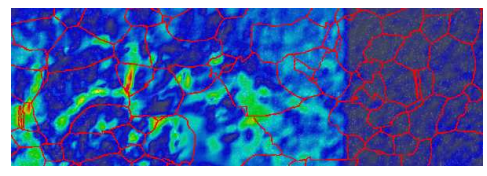

(j) $\mathrm{t}=115 \mathrm{~s}, \mathrm{~T}=T_{\alpha-\gamma}+58 K$

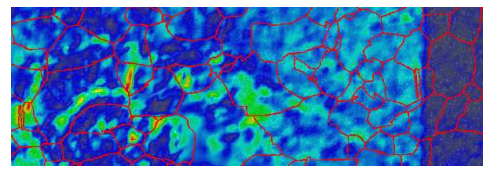

(m) t $=131 \mathrm{~s}, \mathrm{~T}=T_{\alpha-\gamma}+118 K$

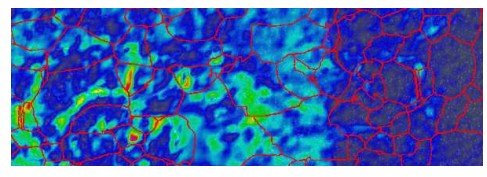

(p) $\mathrm{t}=144.25 \mathrm{~s}, \mathrm{~T}=T_{\alpha-\gamma}+88 \mathrm{~K}$

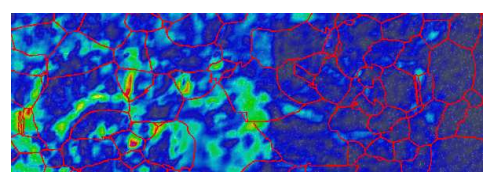

(s) $\mathrm{t}=145.75 \mathrm{~s}, \mathrm{~T}=T_{\alpha-\gamma}+58 K$

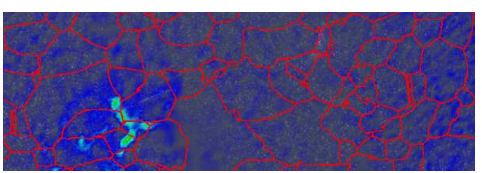

(b) $\mathrm{t}=100 \mathrm{~s}, \mathrm{~T}=T_{\alpha-\gamma}$

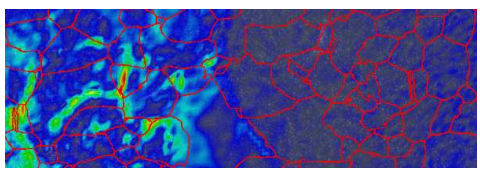

(e) $\mathrm{t}=102 \mathrm{~s}, \mathrm{~T}=T_{\alpha-\gamma}+7 K$

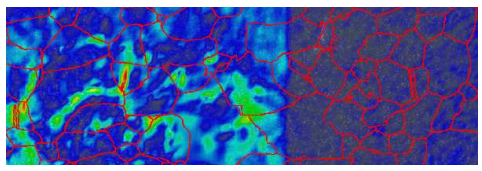

(h) $\mathrm{t}=106.5 \mathrm{~s}, \mathrm{~T}=T_{\alpha-\gamma}+28 K$

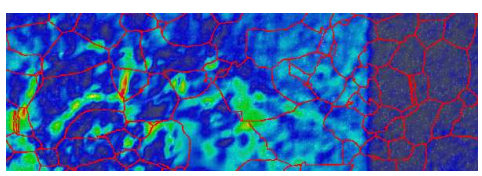

(k) $\mathrm{t}=120.25 \mathrm{~s}, \mathrm{~T}=T_{\alpha-\gamma}+78 K$

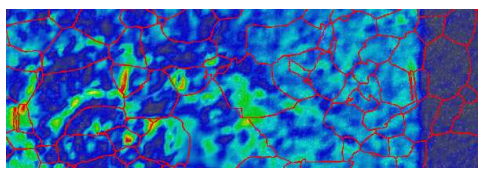

(n) $\mathrm{t}=137.25 \mathrm{~s}, \mathrm{~T}=T_{\alpha-\gamma}+$ $118 K$

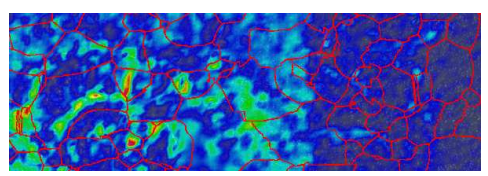

(q) $\mathrm{t}=144.75 \mathrm{~s}, \mathrm{~T}=T_{\alpha-\gamma}+78 K$

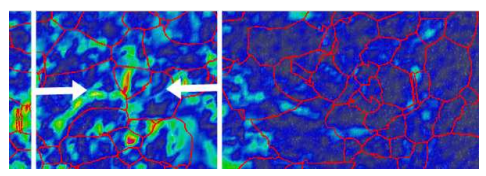

(t) $\mathrm{t}=146.75 \mathrm{~s}, \mathrm{~T}=T_{\alpha-\gamma}+33 K$

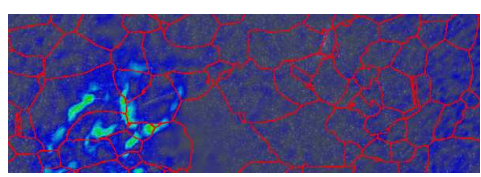

(c) $\mathrm{t}=100.25 \mathrm{~s}, \mathrm{~T}=T_{\alpha-\gamma}+1 K$

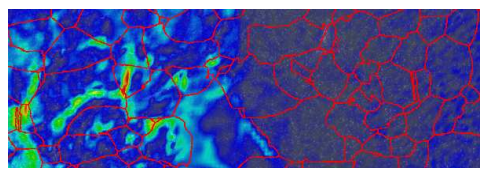

(f) $\mathrm{t}=102.5 \mathrm{~s}, \mathrm{~T}=T_{\alpha-\gamma}+8 K$

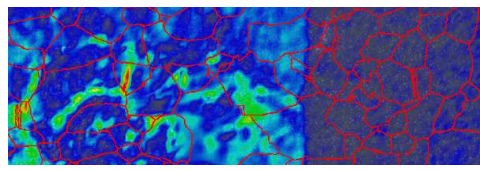

(i) $\mathrm{t}=109.25 \mathrm{~s}, \mathrm{~T}=T_{\alpha-\gamma}+38 K$

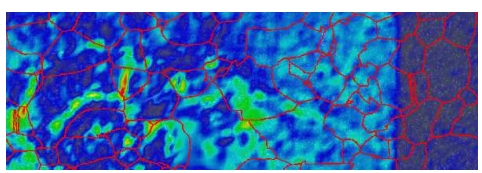

(l) $\mathrm{t}=125.5 \mathrm{~s}, \mathrm{~T}=T_{\alpha-\gamma}+98 \mathrm{~K}$

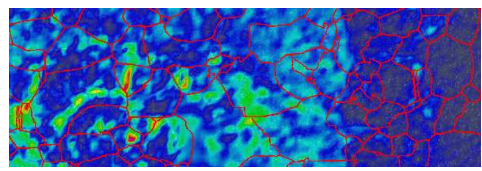

(o) $\mathrm{t}=143.75 \mathrm{~s}, \mathrm{~T}=T_{\alpha-\gamma}+98 K$

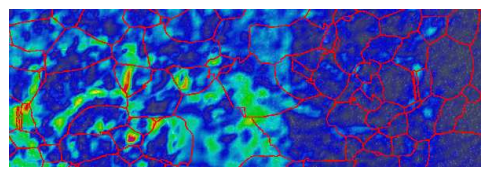

(r) $\mathrm{t}=145.25 \mathrm{~s}, \mathrm{~T}=T_{\alpha-\gamma}+68 K$

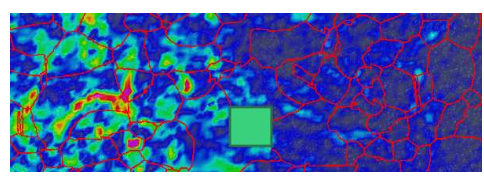

(u) $\mathrm{t}=148 \mathrm{~s}, \mathrm{~T}=T_{\alpha-\gamma}+8 K$

Figure 8: Maximum shear strain evolution (as a percentage) with temperature variations at the centre of the sample represented in the zone shown in Figure 6 

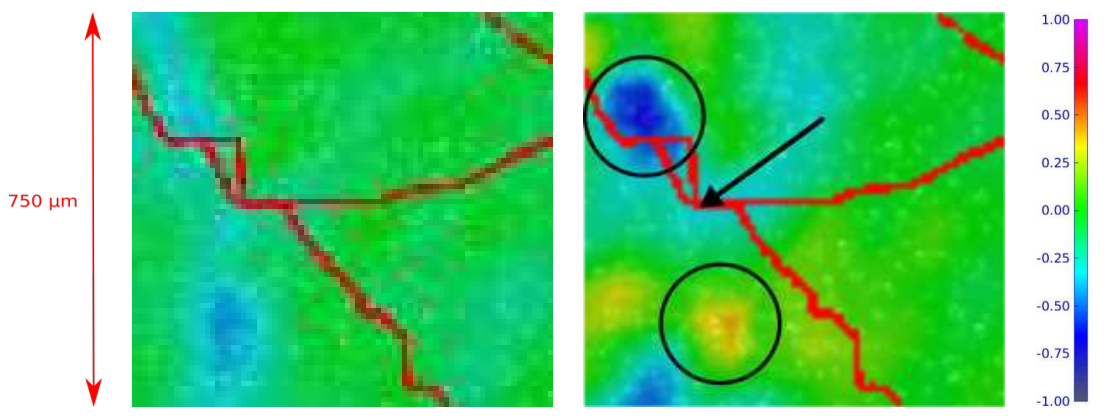

Figure 9: Shear strain field near a triple point before and after the onset of transformation (as a percentage). The studied zone corresponds to the green square in Figure $8 \mathrm{u}$.
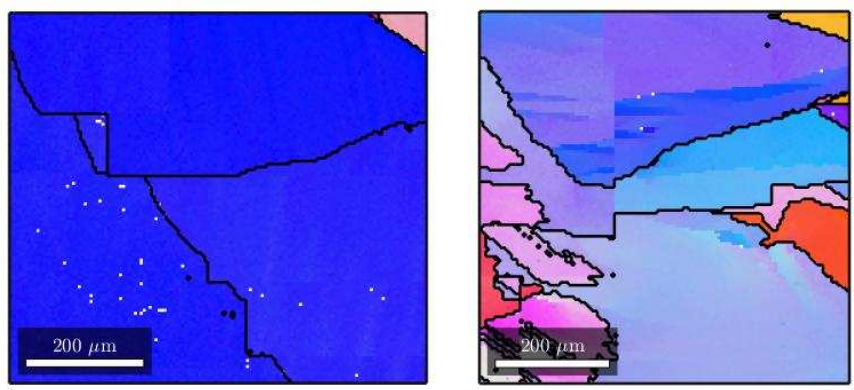

Figure 10: Zoom on the initial (Figure 1) and final EBSD maps in the area of interest.
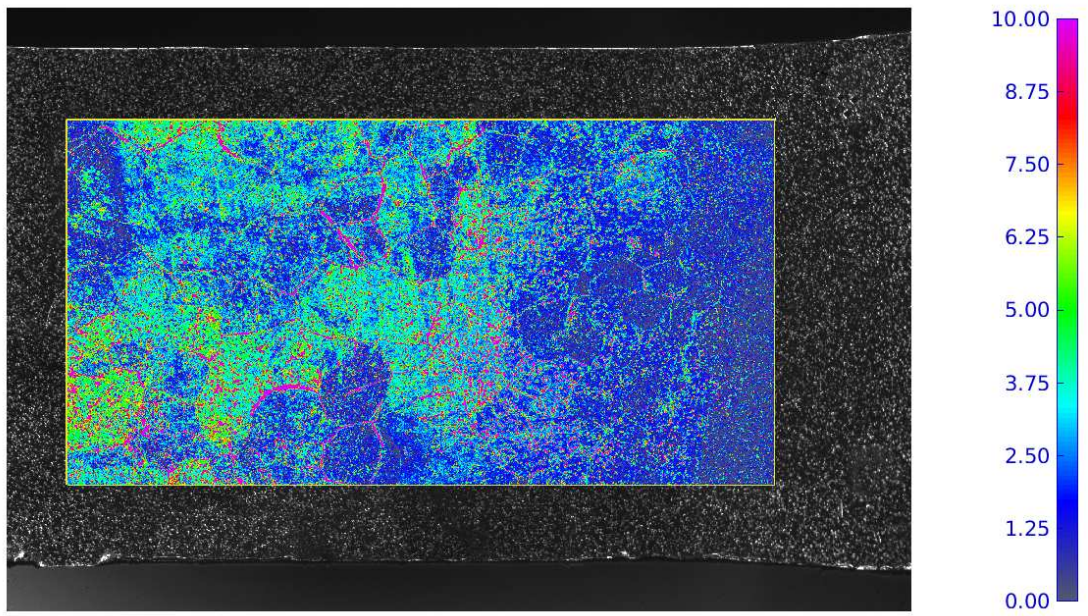

Figure 11: Pixelwise error in gray levels of correlation calculation at the end of heating phase (as a percentage) 


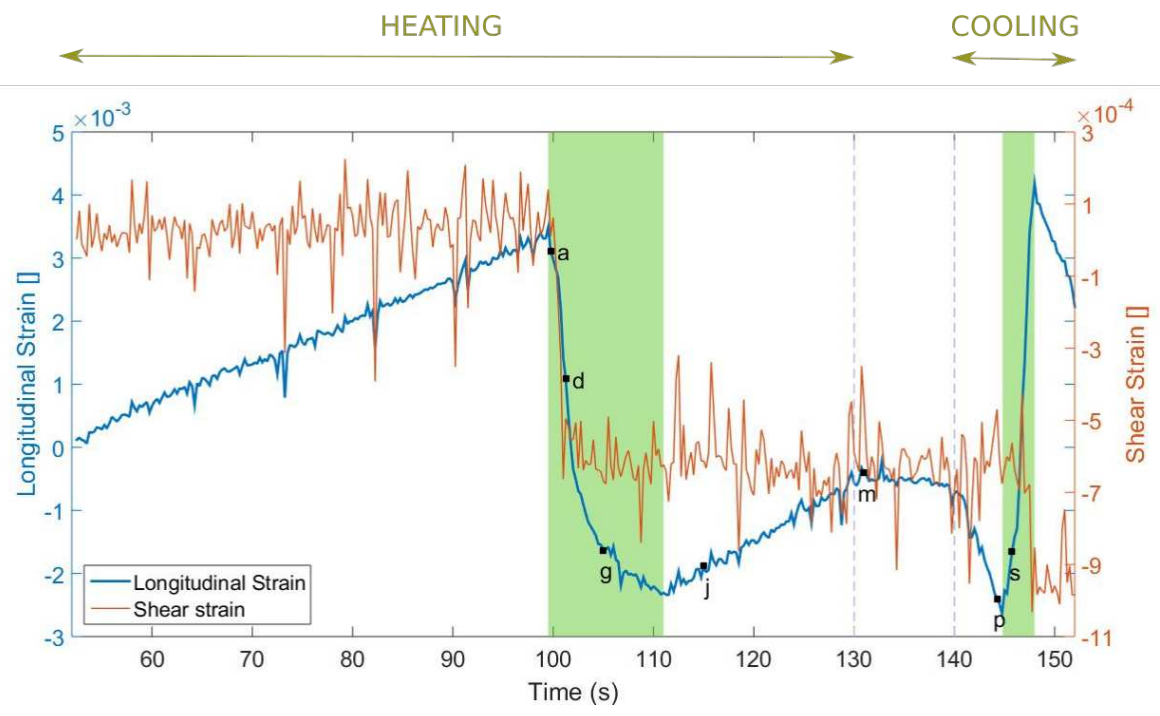

Figure 12: Evolution of longitudinal and shear strains averaged on the whole domain. The letters correspond to the image labels in Figure 8
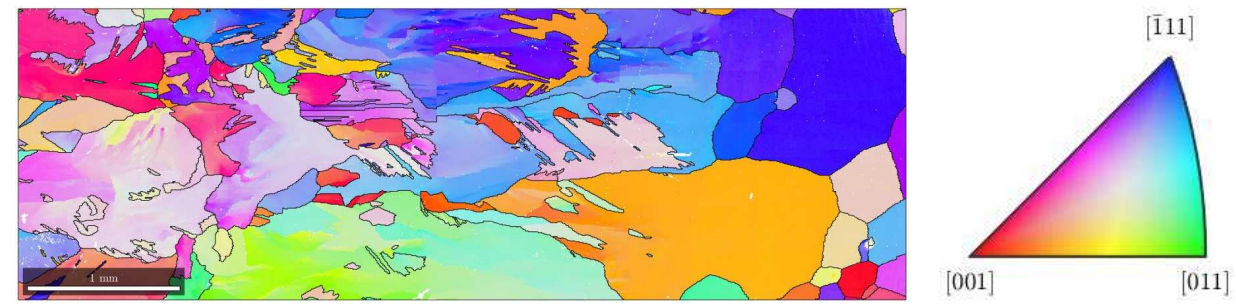

Figure 13: Post mortem EBSD analysis. Grains are colour-coded according to the adjacent key Inverse Pole Figure.

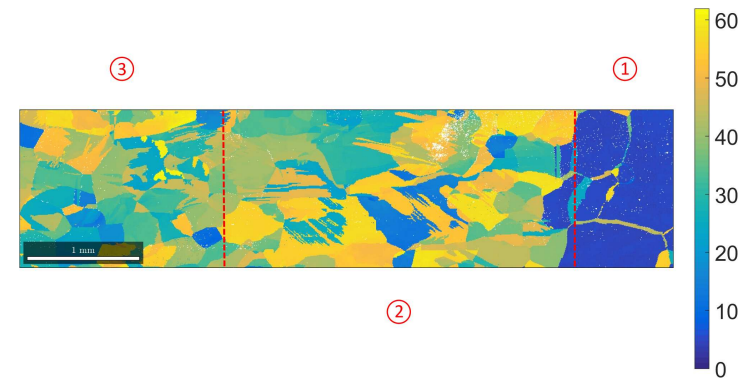

(a) Minimal angles in degrees between initial and final states

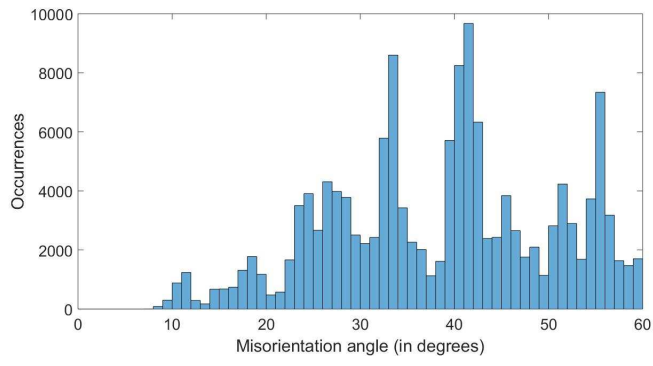

(b) Histogram of minimal angles between inital and final states in zone 2

Figure 14: Orientations evolution between initial and final states 